\title{
A generalization of a Conservativity Theorem for Classical versus Intuitionistic Arithmetic
}

\author{
Stefano Berardi \\ http://www.di.unito.it/ ${ }^{\sim}$ stefano
}

\begin{abstract}
A basic result in Intuitionism is $\Pi_{2}^{0}$-Conservativity. Take any proof $p$ in Classical Arithmetic of some $\Pi_{2}^{0}$-statement (some arithmetical statement $\forall x . \exists y . P(x, y)$, with $P$ decidable). Then we may effectively turn $p$ in some intuitionistic proof of the same statement. In a previous paper [1], we generalized this result: any classical proof $p$ of an arithmetical statement $\forall x \cdot \exists y \cdot P(x, y)$, with $P$ of degree $k$, may be effectively turned into some proof of the same statement, using Excluded Middle only over degree $k$ formulas. When $k=0$, we get the original conservativity result as particular case. This result was a byproduct of a semantical construction. J. Avigad, of Carnegie Mellon University, found a short, direct syntactical derivation of the same result, using H. Friedman's $A$-translation. His proof is included here with his permission.
\end{abstract}

Iniziato a Torino, il 2 Aprile 2003. Ultimo salvataggio: September 2, 2003

\section{Plan of the paper.}

In section 2 we quickly introduce Classical and Intuitionistic Arithmetic, and we state Generalized conservativity. In section 3 we introduce Friedman's $A$-translation, and we explain how it was used to prove $\Pi_{2}^{0}$-conservativity. In section 4 we use Friedman's $A$-translation to prove Generalized Conservativity.

Generalized Conservativity could look uninteresting, from an intuitionistic viewpoint. Indeed, it produces proofs using Excluded Middle, even if only over degree $k$ formulas. We think of it instead as a 
step toward extracting some constructive content from any classical proof, not just from proofs of $\Pi_{2}^{0}$-statements. We defend our claim in section 5 .

\section{Stating Generalized Conservativity}

We quickly recall what Intuitionistic and Classical Arithmetic are.

A Language $L$ for Arithmetic Let $L$ be the first order language including equality and function symbols for 0 (zero), $s$ (successor) and any primitive recursive map. Any other primitive recursive predicate, like $x \leq y$, is introduced through its characteristic maps. We use the connectives $\perp, \wedge, \vee, \rightarrow, \forall, \exists$. We consider $\neg A$ as abbreviating $A \rightarrow$ $\perp$. We call degree of a formula $A$, and we denote it by $\operatorname{deg}(A)$, the maximum number of nested quantifiers in $A$. Degree 0 formulas are propositional formulas. In the intended interpretation, they are all decidable (in fact, primitive recursive).

Axioms and Rules for Arithmetic. Call atomic rule any rule $A_{1}, \ldots, A_{n} \vdash$ $A$, with $A_{1}, \ldots, A_{n}, A$ atomic, and closed under first order substitution. Rules for equality (reflexivity, symmetry, transitivity, compatibility with functions) are atomic. We call induction schema the axiom schema $A[x:=0] \rightarrow \forall x .(A \rightarrow A[x:=s(x)]) \rightarrow \forall x$.A, for all formulas of $L$. We call Excluded Middle of degree $k$, or $\mathrm{EM}_{k}$ for short, the axiom schema $A \vee \neg A$ for all formulas of degree $k$. We call EM the axiom schema $A \vee \neg A$ for all formulas of $L$.

Minimal, Intuitionistic and Classical Logic for L. Rules of First Order Intuitionistic Logic are Rules for Equality, Introduction and Elimination rules for all connectives (rules for $\neg$ are obtained as particular case of rules for $\rightarrow$ ). Minimal Logic is obtained by removing Elimination of $\perp$. Without this rule, we cannot prove $\perp \rightarrow A$ for any $A$, and $\perp$ becomes a propositional constant we know nothing of. Classical Logic is obtained by adding EM to Intuitionistic Logic.

Minimal, Intuitionistic and Classical Arithmetic. They are obtained from, respectively, Minimal, Intuitionistic and Classical Logic, by adding all equational axioms for primitive recursive maps, Induction, and the axiom $\neg(0=S(t))$. ${ }^{1}$ We also call Intuitionistic Arithmetic "Heyting Arithmetic", and Classical Arithmetic "Peano Arith-

\footnotetext{
${ }^{1}$ The axioms $s(t)=s(u) \rightarrow t=u$ and $0=s(t) \rightarrow u=v$ for successor (with $t, u$ any terms of $L$ ) are derivable from equational axioms for predecessor and conditional: $\operatorname{pred}(S(t))=t$ and $\operatorname{if}(0, u, v)=u$, if $(S(t), u, v)=v$.
} 
metic". We denote Minimal, Intuitionistic and Classical Arithmetic respectively by MA, HA, PA. For any theory $T$, we denote by $T \vdash A$ the statement: "the formula $A$ is derivable in $T$ ". By induction over $A$ we may check that MA $\vdash 0=s(t) \rightarrow A$ for all $A$. In Intuitionistic Logic, $\mathrm{EM}_{k}$ proves $\mathrm{EM}_{h}$ for any $h \leq k$ (just add vacuous quantifiers in front of $A$ in $A \vee \neg A$ ). HA proves $x=y \vee x \neq y$ (Excluded Middle for equality) using Induction twice. Then it proves (all formulas in) $\mathrm{EM}_{0}$ out of it. HA cannot prove $\mathrm{EM}_{1}$ instead. A fortiori, HA proves $\mathrm{EM}_{k}$ for no $k>0$.

Replacing $\perp$ by any formula. If $F, A$ are any formulas, call $F[\perp:=$ $A$ ] the result of replacing $\perp$ with $A$ everywhere in $F$ (and possibly renaming bound variables, in order to avoid capture of free variables). In any proof of MA we may replace $\perp$ by any $A$, obtaining still a correct proof. The reason is that the only assumption over $\perp$ in MA is $0=s(t) \rightarrow \perp$, and this axiom becomes $0=s(t) \rightarrow A$, which is provable in MA. Thus, if MA $\vdash F$, then MA $\vdash F[\perp:=A]$ for all formulas A.

We intend to prove the following result:

Theorem 2.1 (Generalized Conservativity) If $\operatorname{deg}(P)=k$, and PA $\vdash$ $\forall x . \exists y . P$, then $\mathrm{HA}+\mathrm{EM}_{k} \vdash \forall x . \exists y \cdot P$.

$\Pi_{2}^{0}$-Conservativity is the statement: if $\operatorname{deg}(P)=0$, and PA $\vdash$ $\forall x . \exists y . P$, then HA $\vdash \forall x . \exists y . P$. It is obtained as particular case, by setting $k=0$, then remarking that $\mathrm{HA}$ proves $\mathrm{EM}_{0}$. A quick way of proving $\Pi_{2}^{0}$-Conservativity result (for Classical versus Intuitionistic Arithmetic) was introduced by H. Friedman. Avigad shown that Generalized Conservativity (of which we had a semantical proof) may be proved using Friedman $A$-translation.

\section{Friedman's $A$-translation}

Call Gödel's $\neg \neg$-translation of $F$ (see [3]) the formula $F\urcorner \neg$, obtained replacing each sub-formula $t=u$ of $F$ by $\neg \neg t=u$, and each subformula $B \vee C$ or $\exists x$. $B$ of $F$, respectively, by $\neg(\neg B \wedge \neg C)$ or $\neg \forall x$. $\neg B$. ${ }^{2}$ For instance, $\left.\perp \neg\right\urcorner=\perp$. Gödel's translations of $\perp \rightarrow F$ and $F \vee \neg F$ are derivable within Minimal Logic. Gödel proved the following:

Theorem 3.1 If PA $\vdash F$, then MA $\vdash F\urcorner$.

\footnotetext{
${ }^{2}$ Actually, we replace $\vee, \exists$ by $\neg \wedge \neg, \neg \forall \neg$, while Gödel replaced them with $\neg \neg \vee, \neg \neg \exists$. However, two choices are intuitionistically equivalent.
} 
The proof is by induction over the proof in PA (see [3]). Gödel's $\neg \neg$ translation is a faithful interpretation of PA in MA. Indeed, if $\operatorname{deg}(F)=$ $k$, then in $\mathrm{HA}+\mathrm{EM}_{k}$ we may prove $\left.\left.F \leftrightarrow F\right\urcorner\right\urcorner$. Thus, if MA $\left.\vdash F\right\urcorner \neg$, then $\mathrm{HA}+\mathrm{EM}_{k} \vdash F$, hence PA $\vdash F$. Summing up, PA $\vdash F$ iff MA $\left.\vdash F\right\urcorner \neg$ iff $\mathrm{HA}+\mathrm{EM}_{k} \vdash F .{ }^{3}$

Fix any formula $A$. Call Friedman $A$-translation of $F$ (see [2]) the formula $F\urcorner \neg[\perp:=A] .{ }^{4}$ For instance: $\left.\left.\perp^{A}=\perp\right\urcorner\right\urcorner[\perp:=A]=\perp[\perp:=$ $A]=A$, while $\left.\left.(t=u)^{A}=(t=u)\right\urcorner\right\urcorner[\perp:=A]=\neg \neg(t=u)[\perp:=A]=$ (by replacing $\neg$ with its definition) $((t=u \rightarrow A) \rightarrow A)$.

We may characterize Friedman $A$-translation as follows. Use $\wedge^{A}, \rightarrow^{A}$ ,$\forall^{A}$ just as an alternative denotation for $\wedge, \rightarrow, \forall$. Define the $A$ negation of $F$, in symbols $\neg^{A} F$, as $F \rightarrow A$. Define $B \vee^{A} C$ as $\neg^{A}\left(\neg^{A} B \wedge \wedge^{A} \neg^{A} C\right)$. Define $\exists^{A} x . B$ as $\neg \neg^{A} \forall^{A} x . \neg^{A} B$. Just by definition unfolding, we may check that $F^{A}$ is obtained by replacing every atom $a$ in $F$ by $a^{A}$, and every connective $c$ in $F$ by $c^{A}$.

We may derive the following Theorem:

Theorem 3.2 (Friedman A-Translation) If PA $\vdash F$, then MA $\vdash F^{A}$ for any $A$.

Proof. If PA $\vdash F$, then MA $\vdash F\urcorner \neg$, and MA $\vdash F \neg \neg[\perp:=A]$ by replacing $\perp$ with $A$. Thus, MA $\vdash F^{A}$.

We may derive $\Pi_{2}^{0}$-Conservativity using $A$-translation. First of all, we remark that we have only to prove $\Sigma_{1}^{0}$-Conservativity, which is the statement: if $\operatorname{deg}(P)=0$, and PA $\vdash \exists y . P$, then HA $\vdash \exists y . P$. The reason is: for any theory $T$ with Introduction and Elimination of $\forall$, we have $T \vdash A$ iff $T \vdash \forall x$. A. Second, we remark that every degree 0 formula $P$, with free variables $\underline{x}=x_{1}, \ldots, x_{n}$ is equivalent in HA to some equation $f(\underline{x})=0$ (just take the primitive recursive characteristic map $f$ of $P$ ). Therefore we may assume that $P$ is an equational statement.

The next step is a curious Lemma, which C. Murphy proposed to call "Friedman Topmost Trick". ${ }^{5}$ Fix any $A=\exists x . P$, with $P$

\footnotetext{
${ }^{3}$ Faithfulness of Gödel's translation might seem to be enough to prove Generalized Conservativity. It is not. If $F=\forall x \cdot \exists y \cdot P$, and $\operatorname{deg}(P)=k$, then $\operatorname{deg}(F)=k+2$. Faithfulness implies: if PA $\vdash F$, then $\mathrm{HA}+\mathrm{EM}_{k+2} \vdash F$. Generalized Conservativity requires, instead: if $\mathrm{PA} \vdash F$, then $\mathrm{HA}+\mathrm{EM}_{k} \vdash F$.

${ }^{4}$ Friedman $A$-translation was originally intended from Intuitionistic to Minimal Logic. To be accurate, therefore, we should call $F^{A}$ "the composition of $A$-translation with Gödel's translation, applied to $F "$. We prefer to call $F^{A}$ directly "the $A$-translation of $F "$, for short.

${ }^{5}$ The proof of this Lemma plays a key role in Continuation Theory.
} 
equational. The Lemma says: if we $A$-translate the negation $\forall x . \neg P$ of $A$ itself, we obtain a statement true in Minimal Logic. If we $A$ translate $A$ itself, we get a statement equivalent to $A$ in Minimal Logic.

Lemma 3.3 (Friedman Topmost Trick) Fix any $A=\exists x . P$, with $P$ equational.

- $(\forall x . \neg P)^{A}$ is derivable in Minimal Logic.

- $A^{A}$ is equivalent to $A$ in Minimal Logic.

Proof.

- We have $(\forall x . \neg P)^{A}=\forall^{A} x . \neg^{A} P^{A}=\forall x .\left(P^{A} \rightarrow A\right)=(P$ is equational):

$$
\text { (1) } \forall x \cdot(((P \rightarrow A) \rightarrow A) \rightarrow A)
$$

Since $A=\exists x . P$, by $\exists$-Introduction we obtain $P \rightarrow A$. We conclude that (1) above is equivalent to $\forall x .(A \rightarrow A)$, hence it is derivable.

- We have $A^{A}=\exists^{A} x \cdot P^{A}=\neg \neg^{A} \forall A x . \neg^{A} P^{A}$. By unfolding the first $\neg^{A}$ we obtain $A^{A}=\left(\left(\forall A x . \neg^{A} P^{A}\right) \rightarrow A\right)=\left((\forall x . \neg P)^{A} \rightarrow A\right)$. By the previous point, $(\forall x . \neg P)^{A}$ is derivable in minimal logic. We conclude that $A^{A}$ is equivalent to $A$ in minimal logic.

$\Pi_{2}^{0}$ - Conservativity is an immediate consequence of Friedman Topmost Trick. Assume we may prove $A=\exists x . P$ in PA, with $P$ equational. Then in MA we may prove $A^{A}$, hence $A$. We conclude HA $\vdash A, \mathrm{Q}$. E. D..

\section{Proving Generalizing Conservativity}

Our goal is now to extend the Conservativity result to $A=\exists x . P(x, y)$, with $\operatorname{deg}(P)=k$, and to $\mathrm{PA}, \mathrm{HA}+\mathrm{EM}_{k}$. Therefore we have to extend Friedman Topmost Trick as follows: in $\mathrm{HA}+\mathrm{EM}_{k}$, we may prove both $(\forall x . \neg P)^{A}$, and $A^{A} \leftrightarrow A$.

Fix any formula $A$, any $k \in N$, any connective $c$ of $L$. The first step is to prove, in $\mathrm{HA}+\mathrm{EM}_{k}$, that if $c$ has all arguments of degree $\leq k$, then the map $B \mapsto B \vee A$ commutes with $c, c^{A}$.

Lemma 4.1 (Commutativity Lemma) Fix any formula A, any $k \in$ $N$. The following equivalences are provable in $\mathrm{HA}+\mathrm{EM}_{k}$, for any $B, C, \forall x . D, \exists x . E$ of degree $\leq k$ : 
- $(\wedge)(B \wedge C) \vee A \leftrightarrow(B \vee A) \wedge^{A}(C \vee A)$

- $(\rightarrow)(B \rightarrow C) \vee A \quad \leftrightarrow(B \vee A) \rightarrow^{A}(C \vee A)$

- $(\forall)(\forall x . D) \vee A \leftrightarrow \forall^{A} x .(D \vee A)$

- $(\vee)(B \vee C) \vee A \quad \leftrightarrow(B \vee A) \vee^{A}(C \vee A)$

- $(\exists)(\exists x . E) \vee A \quad \leftrightarrow \exists^{A} x .(E \vee A)$

\section{Proof}

- $(\wedge)$. By distributivity of $\wedge^{A}=\wedge$ with $\vee$.

- $(\rightarrow)$. $\Rightarrow$. Assume $(B \rightarrow C) \vee A$, in order to prove $(B \vee A) \rightarrow$ $(C \vee A)$. We assume $B \vee A$ and we have to prove $C \vee A$. If $B \rightarrow C$, then $C \vee A$ because we assumed $B \vee A$. If $A$, then $C \vee A$. $\Leftarrow$. Assume $(B \vee A) \rightarrow(C \vee A)$, in order to prove $(B \rightarrow C) \vee A$. We use $B \vee \neg B$, and $C \vee \neg C$. If either $\neg B$ or $C$, we deduce $B \rightarrow C$, and we conclude $(B \rightarrow C) \vee A$. If $B$ and $\neg C$, we deduce $B \vee A$, hence $C \vee A$ by assumption. By $\neg C$ we obtain $A$, and we conclude $(B \rightarrow C) \vee A$.

- $(\forall) . \Rightarrow$. Assume $(\forall x . D) \vee A$, in order to prove $\forall x .(D \vee A)$. $x$ is a bound variable of $\forall x . D$, and we may assume that $x$ is not free in $A$. If $\forall x . D$, then $\forall x .(D \vee A)$. If $A$, then $D \vee A$, and $\forall x$. $(D \vee A)$ because $x$ is not free in the assumption $A . \Leftarrow$. Assume $\forall x .(D \vee A)$, in order to prove $(\forall x . D) \vee A$. We use $(\exists x . \neg D) \vee$ $(\neg \exists x . \neg D)$ and $D \vee \neg D$. Out of them we may intuitionistically derive $(\exists x . \neg D) \vee(\forall x . D)$. If $\exists x . \neg D$, from $\forall x$. $(D \vee A)$ we obtain $A$, then $(\forall x . D) \vee A$. If $\forall x . D$, then $(\forall x . D) \vee A$.

- $(\vee)$. $\Rightarrow$. Assume $B \vee C \vee A$, in order to prove $(B \vee A) \vee^{A}(C \vee A)$. By definition of $\vee^{A}$, we assume $\neg^{A}(B \vee A), \neg^{A}(C \vee A)$, and we have to prove $A$. The new assumptions are, by definition, $(B \vee A) \rightarrow A$ and $(C \vee A) \rightarrow A$. Our thesis, $A$, follows by case reasoning over $B \vee C \vee A$ and Modus Ponens. $\Leftarrow$. Assume $(B \vee A) \vee^{A}(C \vee A)$, that is, $\neg^{A}\left(\neg^{A}(B \vee A) \wedge \neg^{A}(C \vee A)\right)$, in order to prove $B \vee C \vee A$. We use $B \vee \neg B$ and $C \vee \neg C$. If $B$ or $C$, then $(B \vee C) \vee A$. If both $\neg B, \neg C$, we deduce $(B \vee A) \rightarrow A$ and $(C \vee A) \rightarrow A$. By definition, we obtain $\neg^{A}(B \vee A), \neg^{A}(C \vee A)$ and $\neg^{A}(B \vee A) \wedge \neg^{A}(C \vee A)$. Our assumption is the $A$-negation of this latter: we conclude $A$.

- $(\exists)$. $\Rightarrow$. Assume $(\exists x . E) \vee A$, in order to prove $\exists^{A} x .(E \vee A)$. By definition of $\exists^{A}$, we assume $\forall x \cdot \neg^{A}(E \vee A)$ and we have to prove 
$A$. The new assumption unfolds to $\forall x .((E \vee A) \rightarrow A)$. We reason by cases over $(\exists x . E) \vee A . \quad x$ is a bound variable of $\exists x . E$, and we may assume that $x$ is not free in $A$. In the case $\exists x . E$ holds, we first deduce $\exists x .(E \vee A)$. From it and $\forall x .((E \vee A) \rightarrow A)$ we conclude $A$, by $\exists$-Elimination and $x$ not free in $A$. In the case $A$ holds, we conclude $A$ again. $\Leftarrow$. Assume $\exists^{A} x .(E \vee A)$, that is, $(\forall x .(E \vee A \rightarrow A)) \rightarrow A$, in order to prove $(\exists x . E) \vee A$. We use $(\exists x . E) \vee \neg(\exists x . E)$. If $(\exists x . E)$, we conclude $(\exists x . E) \vee A$. If $\neg \exists x . E$, then we intuitionistically deduce $\forall x . \neg E$ and $\forall x .((E \vee A) \rightarrow A)$ from it. By our assumption and Modus Ponens we deduce $A$. We conclude $(\exists x . E) \vee A$.

The next step is to prove, in $\mathrm{HA}+\mathrm{EM}_{k}$ again, that for any formula $A$, and for any formula $F$ of degree $\leq k, F^{A}$ is equivalent to $F \vee A$, no matter what $A$ is.

Lemma 4.2 (Avigad Lemma) If $\operatorname{deg}(F) \leq k$, then $F^{A} \leftrightarrow F \vee A$ in $\mathrm{HA}+\mathrm{EM}_{k}$.

Proof. By induction over $F$.

Assume $F=\perp$. Then the thesis is $A \leftrightarrow \perp \vee A$, derivable in Intuitionistic Logic.

Assume $F$ is any equation. Then $F \vee \neg F$ holds in HA, and the thesis is $((F \rightarrow A) \rightarrow A) \leftrightarrow F \vee A$. $\Rightarrow$. Assume $(F \rightarrow A) \rightarrow A)$. If $F$, then $F \vee A$. If $\neg F$, then $F \rightarrow A$. From our assumption we deduce $A$, then $F \vee A$. $\Leftarrow$. Assume $F \vee A$. If $F$, then $F \rightarrow A$ implies $A$, hence $(F \rightarrow A) \rightarrow A$. If $A$, then $(F \rightarrow A) \rightarrow A$ again.

Assume $F=(B \wedge C),(B \rightarrow C), \forall x \cdot D,(B \vee C), \exists x . E$. Then $F^{A}=\left(B^{A} \wedge^{A} C^{A}\right),\left(B^{A} \rightarrow{ }^{A} C^{A}\right), \forall^{A} x . D^{A},\left(B^{A} \vee^{A} C^{A}\right), \exists^{A} x . E^{A}$. Вy induction hypothesis over $B, C, D, E$, the formula $F^{A}$ is equivalent in $\mathrm{HA}+\mathrm{EM}_{k}$ to, respectively, $\left((B \vee A) \wedge^{A}(C \vee A)\right),\left((B \vee A) \rightarrow^{A}\right.$ $(C \vee A)), \forall^{A} x .(D \vee A),\left((B \vee A) \vee^{A}(C \vee A)\right), \exists^{A} x .(E \vee A)$. By applying the previous Lemma, $F^{A}$ is equivalent in $\mathrm{HA}+\mathrm{EM}_{k}$ to $(B \wedge C) \vee A,(B \rightarrow$ $C) \vee A,(\forall x . D) \vee A,(B \vee C),(\exists x . E) \vee A$. In all cases, what we got is $F \vee A$.

We may now derive a generalization of Friedman Topmost Trick, and generalized Conservativity out of it.

Lemma 4.3 (Generalized Friedman Topmost Trick) Fix any $A=$ $\exists x . P$, with $\operatorname{deg}(P)=k \in N$. 
- $(\forall x . \neg P)^{A}$ is derivable in $\mathrm{HA}+\mathrm{EM}_{k}$.

- $A^{A}$ is equivalent to $A$ in $\mathrm{HA}+\mathrm{EM}_{k}$.

Proof.

- We have $(\forall x . \neg P)^{A}=\forall{ }^{A} x . \neg{ }^{A} P^{A}=\forall x .\left(P^{A} \rightarrow A\right)$. By Avigad's Lemma, this latter is equivalent to $\forall x .(P \vee A \rightarrow A)$ in $\mathrm{HA}+\mathrm{EM}_{k}$. Since $A=\exists x . P$, by $\exists$-Introduction we deduce $P \rightarrow A$ is true. We deduce $P \vee A \rightarrow A$ out of it, then $\forall x .(P \vee A \rightarrow A)$ by $\forall$-introduction. We conclude $(\forall x . \neg P)^{A}$ in $\mathrm{HA}+\mathrm{EM}_{k}$.

- We have $A^{A}=\exists^{A} x \cdot P^{A}=\neg^{A} \forall{ }^{A} x \cdot \neg^{A} P^{A}$. By unfolding the first $\neg^{A}$ we obtain $A^{A}=\left(\left(\forall^{A} x \cdot \neg^{A} P^{A}\right) \rightarrow A\right)=\left((\forall x . \neg P)^{A} \rightarrow A\right)$. By the previous point, $(\forall x . \neg P)^{A}$ is derivable in $\mathrm{HA}+\mathrm{EM}_{k}$. We conclude that $A^{A}$ is equivalent to $A$ in $\mathrm{HA}+\mathrm{EM}_{k}$.

Theorem 4.4 (Generalized Conservativity) If $\operatorname{deg}(P)=k$, and PA $\vdash$ $\forall x . \exists y . P$, then $\mathrm{HA}+\mathrm{EM}_{k} \vdash \forall x . \exists y . P$.

Proof. Assume we may prove $A=\exists x . P$ in PA, with $\operatorname{deg}(P)=k$. Then in MA we may prove $A^{A}$. By $\mathrm{HA}+\mathrm{EM}_{k} \vdash A^{A} \leftrightarrow A$, we conclude $\mathrm{HA}+\mathrm{EM}_{k} \vdash A$, Q.E.D. .

\section{$5 \quad$ Discussing the result}

We claim that using Generalized Conservativity we may find some constructive content in any Classical Arithmetical proof, not just in proofs of $\Pi_{2}^{0}$-statements.

For instance, fix any recursive map $f: N \rightarrow N$, any $a \in N$, and consider the following Minimum Principle $M$ : " $f$ has a minimum point $x "$, in symbols $M=\exists x . \forall y . f(x) \leq f(y) . M$ is a $\Sigma_{2}^{0}$ statement, hence $\Sigma_{1}^{0}$-conservativity does not apply to it. Indeed, $M$ is derivable in PA, but not in HA. ${ }^{6}$ Generalized Conservativity says that we

${ }^{6} M$ is provable in PA. Assume, for contradiction, that $M$ is false (we are using $\mathrm{EM}_{2}$ here). Then, using Classical Logic again, $\forall x \cdot \exists y . f(x)>f(y)$. We will prove, by total induction over $v \in N$, that for all $c \in N$ we have $f(c)>v$. By induction hypothesis, we assume therefore that $f(c)>v^{\prime}$ for all $c \in N$, all $v^{\prime}<v$, in order to prove $f(c)>v$. Then $f(c) \geq v$ for all $c \in N$. By $\forall x . \exists y . f(x)>f(y)$, we deduce that for some $c^{\prime} \in N$ we have $f(c)>f\left(c^{\prime}\right) \geq v$. We conclude $f(c)>v$. A contradiction now follows by selecting a free variable $a \in N$, then choosing $c=a, v=f(a)$, and deducing $f(a)>f(a)$. Q. E. D..

$M$ is not provable in HA. Roughly speaking, the reason is that cannot find $x$, in general, just by computing finitely many values of $f$. 
may turn any proof $p$ of $M$ into some proof $q$ of the same $M$ using only $\mathrm{EM}_{1} . \mathrm{EM}_{1}$ is intuitionistically equivalent to the axiom schema $\exists z \cdot Q(\underline{z}, z) \vee \forall z . \neg Q(\underline{z}, z)$, for any equation $Q . \mathrm{EM}_{1}$ may be imagined as an oracle $\mathcal{O}$. $\mathcal{O}$ takes a list of values for $\underline{z}$, and returns some pair $\langle b, c\rangle$. If $b=1$, then $Q(\underline{z}, c)$ is true, if $b=2$, then $Q(\underline{z}, z)$ is false for all $z$. $\mathcal{O}$ is a $\Delta_{2}^{0}$-map (often a $\Delta_{2}^{0}$-complete map). Using $\mathcal{O}$ as oracle, $q$ finds, for any $a$, some $x$ such that $\forall y . f(x) \leq f(y)$. Thus, $q$ defines some $\Delta_{2}^{0}$-map $\phi(a) \quad(a \in N$ is the free variable we selected in the end of the proof of $p$ ). We claim that $\phi$ is a constructive content for the original proof $p$. For instance, in the case of the proof $p$ we included in the footnote, $\phi$ is recursively defined as follows. Let $\mathcal{O}$ be an oracle for $(\exists y . f(a)>f(y)) \vee(\forall y . f(a) \leq f(y))$, and $\mathcal{O}(a)=\langle b, c\rangle$. If $b=1$ (and $f(a)>f(c)$ ), then $\phi(a)$ is $\phi(c)$. If $b=2$ (and $f(a) \leq f(y)$ for all $y)$, then $\phi(a)$ is $a$. $\phi(a)$ is always a minimum point for $f$.

In fact, the definition of $\phi$ reflects the ideas in $p . \phi$ uses the oracle $\mathcal{O}$ to produce some decreasing chain $f(a)>f\left(a^{\prime}\right)>f\left(a^{\prime \prime}\right) \ldots$, terminating because $N$ is well-founded. When the chain stops, it stops in some $c$ such that $f(c) \leq f(y)$ for all $y$. $\phi$ is well-defined in Classical Arithmetic. The obvious objection against a constructive use of $\phi$ is that we cannot compute $y=\phi(x)$. But we may think of $\phi(x)$ as a formal notation, which we are able to turn into a real integer whenever we used it to solve a concrete problem. Suppose for instance we have an inequality $f(t) \leq f(t+27)$ (This example is due to T. Coquand). We may solve it using any minimum point $y=\phi(0)$ of $f$. We define in this way a proof of a simple existential statement $\exists t . f(t) \leq f(t+27)$ in Classical Arithmetic. Using $\Sigma_{1}^{0}$-conservativity, we may turn it into some constructive proof of the same result, effectively defining some $t \in N$ such that $f(t) \leq f(t+27)$. After a careful work over the classical proof (not to be included here) we conclude that the definition of $t$ is the following variant of the definition of $\phi$. We look for the first point in which the succession $f(0)>f(27)>f(27+27)>f(27+27+27)>\ldots$ stops decreasing (for the first $27 i$ such that $f(27 i) \leq f(27 i+27)$ ). Definition of $t$ retains ideas from the definition of $\phi(0)$ (but the oracle $\mathcal{O}$ of $\phi$ is now gone).

In general, we claim: by $\Sigma_{1}^{0}$-conservativity, we may use $\Delta_{k}^{0}$-maps in constructive reasoning, to describe ideas in classical proofs of $\Sigma_{k}^{0}$ statements. This is because we may eventually get rid of them when proving $\Sigma_{1}^{0}$-statements. We also have a more elaborate argument. In a previous paper [1] we defined an intuitionistic model $\mathcal{N}$ of $\Delta_{k}^{0}$-maps. 
Thus, it is sound to use $\Delta_{k}^{0}$-maps in intuitionistic reasoning.

The role of Generalized Conservativity is to provide a bound to the complexity of non-recursive maps we may have to use. Suppose we have a classical arithmetical proof $p$ of a $\Sigma_{k}^{0}$-statement. By Generalized Conservativity we may describe the ideas in $p$ using only $\Delta_{k}^{0}$-maps. The bound $k$ is independent from the degree of Excluded Middle we use in $p$. It only depends on the degree of the conclusion of $p$. In the previous example, a proof $p$ of a $\Sigma_{2}^{0}$-statement using $\mathrm{EM}_{2}$ may be turned into a proof $q$ using only $\mathrm{EM}_{1}$. The constructive content of $q$ is then some $\Delta_{2}^{0}$-map $\phi$. Whenever we use $A$ as a Lemma in a proof of some $\Sigma_{1}^{0}$-statement $\exists x . P(x)$, then by $\Sigma_{1}^{0}$-conservativity we may use some suitable recursive variant $\phi^{\prime}$ of $\phi$ to compute $x$.

\section{References}

[1] Stefano Berardi. Classical Logic as Limit II. Technical Report, University of Torino, June 2002. For the proof of Generalized Conservativity see page 48, Faithfulness Theorem, point 2. Web adress: http://www.di.unito.it/ stefano/BerardiClassicalLogicAsLimit-II.rtf

[2] Harvey Friedman. Classically and Intuitionistically Provably Recursive Functions. In Scott, D. S. and Muller, G. H. Editors, Higher Set Theory, Volume 699 of Lecture Notes in Mathematics, Springer Verlag (1978), pp. 21-28.

[3] Kurt Gödel. On Intuitionistic Arithmetic and Number Theory. In Davis M., Editor, The Undecidable, Raven Press (1965), pp. $75-81$.

[4] Chetan Murphy. Extracting Constructive Content from Classical Proofs. Ph.d. Thesis, Cornell University, August 1990, pp 54-59. 